\title{
SINERGIA
}

REVISTA DO INSTITUTO DE CIÊNCIAS ECONÔMICAS, ADMINISTRATIVAS E CONTÁBEIS (ICEAC)

\section{QUALIDADE DE VIDA NO BRASIL: UMA ANÁLISE FATORIAL COM ENFOQUE AOS MUNICÍPIOS DE FRONTEIRA E NÃO FRONTEIRA MARÍTIMA}

TAMIRES MARTINS RAMOS* RAQUEL PEREIRA PONTES*

RESUMO BLANCA LILA GAMARRA MOREL ${ }^{* *}$

\begin{abstract}
Este estudo tem como principal objetivo analisar o Índice de Qualidade de Vida (IQV) dos municípios brasileiros por meio da Análise Multivariada. Para isso, foi necessária a criação desse índice, que considerou os escores fatoriais das dimensões latentes descobertas pela Análise Fatorial Exploratória. A relevância desse novo índice se deve ao fato de que ele possibilita identificar, dentre os 5565 municípios brasileiros, aqueles que apresentam melhor ou pior qualidade de vida - considerando as dimensões ambiental, social e econômica. Além disso, este trabalho tem o intuito de verificar a discrepância de qualidade de vida entre as zonas costeiras (fronteira marítima) e não costeiras (não fronteira marítima) no País. Os resultados obtidos apresentaram seis fatores comuns e um IQV médio de 41,91 para o Brasil como um todo. Ademais, com uma pequena diferença, municípios da Zona Costeira apresentaram um IQV maior quando comparado com os municípios não costeiros.
\end{abstract}

Palavras-chave: Qualidade de vida; Índice; Análise Multivariada; Análise Fatorial Exploratória.

\section{ABSTRACT}

The main objective of this study is to analyze the Quality of Life Index of the Brazilian Municipalities through the Multivariate Analysis. For this, it was necessary to create this index, which considered the factorial scores of the latent dimensions discovered by the Exploratory Factor Analysis. The relevance of this new index is due to the fact that it makes possible to identify, among the 5565 Brazilian municipalities, those that present better or worse quality of life - considering the environmental, social and economic dimensions. In addition, this work aims to verify the quality of life discrepancy between the coastal areas (maritime border) and non-coastal (non-maritime border) in the country. The results obtained presented six common factors and an average IQV of 41.91 for Brazil as a whole. In addition, with a small difference, municipalities in the Coastal Zone presented higher IQV when compared to non-coastal municipalities.

Keywords: Quality of life; Index; Multivariate analysis; Factor analysis

Recebido em: 17-09-2020 Aceito em: 15-01-2021

\section{INTRODUÇÃO}

Segundo a Organização das Nações Unidas (ONU, 2013), um país com alto crescimento econômico nem sempre é um país bem desenvolvido, isso porque crescimento e desenvolvimento econômico possuem significados distintos. $\mathrm{O}$ crescimento econômico está relacionado à capacidade produtiva, que, através do Produto Interno Bruto (PIB), pode-se mensurar a produção de um determinado país ou de uma determinada região. Já o desenvolvimento econômico está relacionado à melhoria da qualidade de vida da população.

No contexto do desenvolvimento econômico, índices pertinentes a serem estudados são: o Índice de Desenvolvimento Humano (IDH) - índice responsável por ponderar indicadores de renda, saúde, educação e expectativa de vida -, com o qual se pode medir o bem-estar dos indivíduos; e o Índice de Gini - um dispositivo que serve para aferir o grau de concentração de renda em um determinado grupo. Esse último índice consegue medir a diversidade de renda, expondo se há uma pequena ou grande diferença entre os mais pobres e os mais ricos em uma determinada região ou país.

Sabe-se que indivíduos com rendas mais elevadas conseguem alcançar melhores condições de bem-

\footnotetext{
Bacharel em Ciências Econômicas pela Universidade Federal do Rio Grande.

" Doutora em Economia Aplicada pelo Programa de Pós-Graduação do Departamento de Economia Rural da UFV. Mestra em Economia Aplicada pelo Programa de Pós-Graduação em Economia Aplicada da FURG (2016). Bacharel em Ciências Econômicas pela Universidade Federal do Rio Grande - FURG (2011). E-mail: raquel_sjn@hotmail.com

D** Doutorado e Mestrado em Economia do Desenvolvimento pela Pontifícia Universidade Católica do Rio Grande do Sul - PUCRS. Professora do Instituto de Ciências Econômicas, Administrativas e Contábeis da Universidade Federal do Rio Grande (FURG).
} 
estar e metas desejáveis de vida. Desse modo, depreende-se que o crescimento da renda tem extrema importância para o aprimoramento das condições de vida das pessoas, pois possibilita um maior acesso a bens e serviços que, até então, eram escassos e, consequentemente, isso propicia uma melhoria na qualidade de vida.

De acordo com o Relatório de Desenvolvimento Humano para a América Latina e Caribe, de 2016 (PNUD, 2016), mais de 72 milhões de pessoas saíram da pobreza e, aproximadamente, 94 milhões entraram na classe média nos países latino-americanos e caribenhos em 2003. Isso foi possível, pois houve um Progresso Multidimensional no qual esses países vivenciaram um processo de mudança histórica que propiciou mudanças significativas que levaram a uma melhora do bem-estar da população com relação a fatores além da renda.

Sendo assim, pode-se dizer que o crescimento econômico é analisado de modo quantitativo, enquanto o desenvolvimento econômico tem por objetivo medir algumas dimensões que não são capturadas pela variação no Produto Interno Bruto. Assim, este último remete a uma forma de avaliar a situação econômica de uma determinada região que abrange o crescimento da produção da economia. A medida do desenvolvimento econômico é relevante, dado que, para melhorar a qualidade de vida dos indivíduos, é necessário analisar outros aspectos sociais, ambientais, políticos, culturais e econômicos.

No Brasil, o IDH apresentou um índice de 0,699 no ano de 2010, ocupando o 73ํlugar no ranking mundial e, quando ajustada a desigualdade, o índice é menor ainda, 0,509 (PNUD, 2010). Em 2015, a PNUD (PNUD, 2015) apresenta um IDH com uma mudança metodológica, com um índice de 0,755 para o Brasil, com destaque positivo para o aumento da expectativa de vida, de anos de escolaridade esperado e da renda nacional bruta per capita, se posicionando em 75일. Jugar. em 2016 (PNUD, 2017), o Brasil ocupa a 79º posição com o mesmo índice de $2015(0,755)$ e com um ajuste da desigualdade para 0,561, com aumento da expectativa de vida e da média de anos de escolaridade, mas com queda da renda nacional bruta per capita quando comparado com 2015. Fora esse cenário de baixo progresso do IDH, há, ainda, grandes disparidades entre municípios, estados e regiões brasileiras.

Ademais, também, pode existir uma desigualdade entre as regiões de fronteira marítima e não marítima (HENDERSON et al., 2018; MOREL, 2018 e RAPPAPORT e SACHS, 2013). Isso pois o Brasil começou a se desenvolver economicamente na região litorânea do País, com a ocupação dos colonizadores nesta área, devido à facilidade do transporte e do comércio através do oceano atlântico. Além do que, a ocupação, nessa região, era importante para a defesa do imenso território brasileiro, visando impedir o acesso de outros países inimigos em busca de novos territórios. Atualmente, outros fatores explicam o maior número da população em regiões costeiras como fenômenos climáticos (por exemplo, mais chuva em regiões litorâneas na região nordeste do País), interesse recreativo, energético, ambiental, tecnológico, científico, entre outros (BRASIL, 2019).

Dentro desse contexto, o presente trabalho busca estudar o nível da qualidade de vida dos municípios brasileiros relacionado ao âmbito econômico, social e ambiental. Também, este estudo tem o intuito de verificar as diferenças de qualidade de vida entre as zonas costeiras e não costeiras no País. Para isso, este estudo utilizará o método da Análise Multivariada, com o objetivo de encontrar quais fatores que melhor explicam a qualidade de vida dos municípios brasileiros. Ainda, aplica-se o método de Análise Fatorial, a fim de simplificar os dados e criar um índice, o (IQV) Índice de Qualidade de Vida.

Com isso, esse estudo propõe contribuir para a literatura de desenvolvimento econômico e bem-estar com o estudo da qualidade de vida dos municípios brasileiros, analisando, também, a diferença entre municípios de fronteira ou não marítima, com o método de Análise Multivariada, gerando um índice a partir dos escores gerados da Análise Fatorial. Ademais, o estudo, também, busca contribuir com os formuladores de política pública, apresentando informações sobre os fatores que mais explicam a qualidade de vida no Brasil. Os principais resultados demonstraram que o nível de IQV médio para os municípios da Zonas Costeira foi de 43,79; para os municípios da Zona Não Costeira, de 41,76 e a média do IQV, para o Brasil, foi de 41,91. Os cinco melhores municípios em termos de IQV foram: São Caetano do Sul $(72,03)$, Vitória (71,94), Nova Lima $(71,39)$, Florianópolis $(70,80)$ e Niterói $(70,73)$. E os cinco piores municípios com relação ao IQV foram: Roteiro $(21,06)$, Branquinha $(21,53)$, Cuité de Mamanguape $(22,15)$, Olivença $(23,03)$ e São José do Piauí $(24,18)$.

Na literatura internacional, importantes estudos trabalharam com o tema qualidade de vida, como, por exemplo, Blomquist, Berger e Hoehn (1981), que analisaram novas estimativas para a qualidade de vida urbana para condados e áreas metropolitanas dos Estados Unidos, ponderando diferentes amenidades dentro e entre áreas urbanas. Slottje, 1991, através de uma análise multidimensional, mede a qualidade de vida de 126 países, incorporando fatores indicados por Amartya Sen. Mais recentemente, Distaso (2007) calculou um índice multidimensional de sustentabilidade para analisar o bem-estar e/ou qualidade de vida nos países da União Europeia, buscando aplicar a teoria do bem-estar de Sen e aplicando o conceito de desenvolvimento humano sustentável, Lambert et al. (2014) concluiu que a qualidade de vida a partir da relação desse com a quantidade de energia disponível e constataram que há uma alta correlação entre o 
índice de energia e o alto padrão de vida e do Carvalhal Monteiro, Pereira e Costa (2019) apresentam diferentes formar de medir o índice de qualidade de vida, como uma abordagem alternativa com base no algoritmo de agrupamento considerando as dimensões do indicador do bem-estar da OCDE.

$\mathrm{Na}$ literatura brasileira, Herculano (1998) revisou estudos que debateram sobre a qualidade de vida, principalmente, em países desenvolvidos e forneceu definições para uso de clusters e de índices. Nahas (2001) estudou a experiência, na capital Belo Horizonte, da construção de um Índice de Qualidade de Vida Urbana (IQVU) e observou a necessidade de aperfeiçoamentos, como a inclusão de mais indicadores ecológicos. Kran e Ferreira (2006) analisaram a qualidade de vida na cidade de Palmas (Estado de Tocantins), por meio de uma análise da habitação e seu entorno, a partir do qual obtiveram o resultado que, ao mesmo tempo que há uma expansão no município de área comercial e residencial, há um crescimento de núcleos carentes. Umbelino (2007) averiguou o Índice de Qualidade de Vida Humana (IQVH) nas regiões metropolitanas brasileiras e evidenciou que o índice melhorou entre 1991 e 2000, mas a desigualdade também aumentou.

Mais recentemente, Morel (2018) analisou o nível de qualidade de vida entre municípios costeiros e não costeiros do Brasil e, através do método de Propensity Score Matching, constatou concentração econômica na área costeira, mas maior desenvolvimento nos municípios não costeiros quando se trata de aspectos ambientais e sociais. Viana et al. (2019) estudaram a qualidade de vida no meio rural brasileiro para o caso do sertão de Alagoas e obtiveram como resultado que o que mais explica a variância da qualidade de vida é o consumo proteico e calórico. Ademais, Kruger, de Oliveira e dos Santos (2020) fizeram uma análise multidimensional da qualidade de vida dos municípios do Rio Grande do Sul e constataram maior índice na cidade de Porto Alegre e menor índice na cidade de Passa Sete.

Além dessa breve introdução, este artigo está organizado da seguinte forma: no segundo capítulo, temse a apresentação de um breve referencial teórico; a descrição do método e dos dados é realizada no terceiro capítulo; os resultados e discussões são apresentados no capítulo quatro e, por fim, têm-se as conclusões.

\section{REFERENCIAL TEÓRICO}

Sen (1989) salienta que uma grande preocupação do desenvolvimento humano está relacionada com a pobreza, estando ela presente tanto nos países desenvolvidos quanto nos que estão em processo de desenvolvimento. Em 1990, o Relatório de Desenvolvimento Humano do Programa das Nações Unidas para o Desenvolvimento (PNUD, 1990) considerou desenvolvimento humano como sendo o processo de expansão da possibilidade de escolha das pessoas, estando, entre essas escolhas, uma vida saudável e acesso à educação e à saúde. Em outras palavras, pode-se dizer que a conquista da ampliação das possibilidades de escolha é sinal de um aumento significativo do nível de bem-estar.

Para Midgley (1995), desenvolvimento econômico tem uma acepção de bem-estar, o que se entende como um círculo virtuoso, ou seja, uma melhoria da renda das pessoas e do nível de educação propiciam melhores condições de saúde e de moradia, o que, por sua vez, leva a um aumento do nível de bem-estar. Contudo, as divergências no conceito de desenvolvimento são mais comuns de serem associadas com uma mudança econômica.

Uma explicação completa de desenvolvimento abrange, além da melhoria de indicadores econômicos e sociais, o meio ambiente. Um país ou uma determinada região pode crescer economicamente e, mesmo assim, não possuir uma qualidade de vida satisfatória; pode ter acesso à moradia ou à educação e não possuir um ar saudável para respirar, pois, com o passar do tempo, o crescimento econômico tende a acabar com os recursos produtivos devido a sua utilização descontrolada.

A preservação do meio ambiente pode ser vista como uma forma de assegurar o cumprimento dos direitos humanos, dado que, na medida em que ocorre um dano ao meio ambiente, haverá, por sua vez, invasão a outros direitos fundamentais, tais como, a vida, a saúde, o bem-estar, dentre outros tantos.

A dimensão e a austeridade dos impasses ambientais que a população vem sofrendo, nos últimos anos, concedem à própria Economia a necessidade de explorar e revisar a questão ambiental, bem como buscar maneiras de aliar desenvolvimento econômico com proteção ambiental. Isso porque meio ambiente e desenvolvimento não são desafios individuais, pois, inegavelmente, estão interligados.

O desenvolvimento não se sustenta se a base dos recursos ambientais se degrada. Por sua vez, o meio ambiente não pode ser conservado se o crescimento econômico não considerar as possíveis consequências de uma destruição ambiental global.

A problemática, aqui exposta, é algo a ser bem analisado, uma vez que qualidade de vida é algo muito complexo, de modo que aquilo que é satisfatório para um indivíduo pode não ser para outro. A definição de qualidade de vida pode ser associada ao bem-estar e ao desenvolvimento econômico, pois aponta o nível das condições básicas do ser humano, condições essas que englobam o bem-estar físico, mental, psicológico e emocional, podendo ser relacionado, também, com a saúde e educação.

Segundo Nussbaum e Sen (1996), a qualidade de vida está associada às capacitações e ao 
funcionamento. Conforme os autores, a vida humana é baseada em um conjunto de "saber e ser", que pode ser nomeado, também, como funcionamentos e está interligado com a avaliação da qualidade de vida.

Gonçalves e Vilarta (2004) dissertam qualidade de vida pelo modo como as pessoas vivem, pela maneira que sentem e compreendem seu dia a dia, abrangendo, assim, saúde, educação, transporte, moradia e trabalho. Mas isso é relativo pois necessita das condições históricas, ambientais e socioculturais de um determinado grupo. Qualidade de vida não se limita no tempo de vida que as pessoas possam ter, mas na interpretação em que elas dão a essas condições e à maneira com que as vive. Para a autora, o termo está relacionado ao significado que se dá às condições objetivas da vida.

Vale ressaltar que, por existirem diversas formas de definição sobre qualidade de vida, escolher apenas uma delas parece indevido, pois esse, ainda, é um campo em processo de definição de conceitos. A pontuação desses autores exibe uma noção que demanda a humanização deste conceito e dos avanços científicos e tecnológicos na área de saúde e bem-estar da sociedade. Tornar humana a saúde e a qualidade de vida não significa, somente, acrescentar anos às vidas dos sujeitos, mas acrescentar vida aos anos (FLECK et al., 1999). Isto é, não basta aumentar a expectativa de vida, mas aprimorar a qualidade dos anos vividos.

\section{METODOLOGIA E DADOS}

\subsection{Método da Análise Multivariada}

Este estudo aplica o método de Análise Multivariada, que pode ser definida como o grupo de estatísticas que possibilitam a análise simultânea dos dados para um ou mais conjuntos de indivíduos (populações ou amostras) descritos por mais de duas variáveis correlacionadas entre si. Embora, em algumas situações. seja proveitoso analisar cada variável isoladamente, percebe-se que, devido à complexidade das dimensões estudadas e por haver inter-relação entre as variáveis, é importante realizar uma análise paralela de todas as variáveis.

\subsubsection{Análise Fatorial}

A Análise Fatorial (AF) foi criada em 1904 por Charles Spearman, um psicólogo americano que, ao realizar um teste em algumas salas de aula, descobriu o fator básico de inteligência geral; o Fator G. A AF nada mais é que uma técnica de Análise Multivariada que tem como principal objetivo minimizar a quantidade de dados analisados, utilizando um número reduzido de informação, definindo, assim, um conjunto de dimensões latentes comuns, denominadas de fatores que, posteriormente, irão explicar as variáveis originárias.

A Análise Fatorial pode ser Exploratória ou Confirmatória. A Exploratória é caracterizada pelo fato de não impor ao pesquisador o conhecimento pregresso da relação de dependência das variáveis, ou seja, parte de um conhecimento empírico. Na Análise Fatorial Exploratória (AFE), o pesquisador estuda, compreende e constata uma relação entre as variáveis a partir do resultado obtido na AF. Já na Análise Fatorial Confirmatória (AFC), o pesquisador parte de um conhecimento sólido de uma estrutura de relacionamento entre as variáveis. A partir disso, a AFC busca confirmar se a teoria que ampara esse relacionamento das variáveis está ou não correta.

É relevante salientar que se deve empregar variáveis que apresentem um padrão, pois uma discrepância, na unidade de medida, pode ocasionar uma oscilação nos dados.

Por este motivo, de acordo com Mingoti (2013, p,113), parte-se de uma matriz de correlação, tendo o seguinte modelo:

$$
\begin{gathered}
Z_{1}=l_{11} F_{1}+l_{12} F_{2}+\cdots+l_{1 m} F_{m}+\varepsilon_{1} \\
Z_{2}=l_{21} F_{1}+l_{22} F_{2}+\cdots+l_{2 m} F_{m}+\varepsilon_{2} \\
\cdots \\
Z_{p}=l_{p 1} F_{1}+l_{p 2} F_{2}+\cdots+l_{p m} F_{m}+\varepsilon_{p}
\end{gathered}
$$

A equação 2 demonstra o modelo da equação 1 em notação matricial:

$$
D(X-\mu)=L F+\varepsilon
$$

$\left.Z_{i}=\left[\left(X_{i}-\mu_{i}\right)\right] / \sigma_{i}\right]$, sendo $Z$ as variáveis padronizadas, $(\mathrm{X}-\mu)$ é o vetor $p \times 1$ de elementos $X_{i}-\mu_{i}$, em que, $i=1,2, \ldots, p, X$ é o vetor de variáveis originais, e $\mu$ é o vetor de médias, Lé a matriz $p \times m$ de cargas fatoriais (correlação entre variáveis originais e fatores), e $\mathrm{F}$ é o vetor $m \times 1$ de fatores comuns (fatores que influenciam duas ou mais variáveis) e $\varepsilon$ é o erro (MINGOTI, 2013). Segundo Lima (2017, p. 41), neste modelo, o fator comum, juntamente com o fator específico (influencia somente uma única variável), e o erro podem explicar a variação total de uma variável. Sendo assim, variação total é igual à comunalidade (fator comum), 
mais a unicidade (fator específico e o erro).

O modelo da Análise Fatorial Exploratória é ortogonal e segue algumas pressuposições (MINGOTI, 2013, p. 102-103): $E\left[F_{j}\right]=0$, ou seja, todos os fatores têm média zero; $\operatorname{Var}\left[F_{m x 1}\right]=I_{m x m}$, os fatores não são correlacionados e têm variância igual a $01 ; E\left[\varepsilon_{p x 1}\right]=I$, os erros têm médias iguais a zero; $\operatorname{Var}\left[\varepsilon_{j}\right]=\Psi_{p x p}$, ou seja, os erros não são correlacionados entre si e têm variâncias diferentes e $E\left[\varepsilon_{p x 1}\right]=0$, isto é, os vetores do erro e dos fatores não são correlacionados.

O modelo AFE pode ser aplicado à rotação de fatores, que se baseia na rotação dos eixos ordenados, alterando as cargas fatoriais, com a finalidade de proporcionar uma melhor interpretação destas e, também, de outras denominações das dimensões latentes.

Por ser uma forma mais simples de rotação, na qual os eixos são mantidos em 90 graus (HAIR et al., 2009), neste estudo, aplica-se a rotação ortogonal pelo método varimax. Logo, são estimados os escores fatoriais que caracterizam o valor de cada fator para cada observação da amostra.

\subsection{2 Índice de Qualidade de Vida (IQV)}

O IQV é calculado por meio de uma média ponderada dos escores fatoriais, em que cada fator é ponderado pela explicação da variância total relacionada a cada fator, conforme é demonstrado na equação 3:

$$
I Q V_{\text {Fatorial }_{i}}=\frac{\sum_{j=1}^{r} \delta j F i j}{\sum_{j=1}^{r} \delta j}, i=1,2, \ldots, n \text { e } j=1,2, \ldots, r
$$

$I Q V_{\text {Fatorial }_{i}}$ é o Índice de Qualidade de Vida $i, \delta j$ é a raiz característica relacionada com o fator $j$,e $F i j$ é o escore fatorial j para a qualidade de vida $i, n$ é o número de observações e $r$ o número de fatores. Com o objetivo de impedir que escores fatoriais altos positivos e/ou negativos sejam criados, é de suma importância que haja uma modificação para que se mantenham no primeiro quadrante:

$$
F_{i j}^{*}=\frac{F_{i j}-F_{j \min }}{F_{j \max }-F_{j \min }}
$$

Analisa-se que o $F_{i j}{ }^{*}$ é o escore fatorial modificado no fator $j$ na qualidade de vida $i, F_{i j}$ o escore fatorial do fator $j$ na qualidade de vida $i, F_{\text {jmin }}$ é o menor valor do j-ésimo escore fatorial, e $F_{j \text { max }}$ é o maior valor do jésimo escore fatorial. Com esse método, todos os escores fatoriais permanecem no intervalo entre zero e um.

Por fim, é realizado um teste de diferença de médias entre os municípios de fronteira (Zona Costeira) e não fronteira marítima (Zona Não Costeira) e, logo após, o mesmo teste é realizado, mas considera-se, apenas, as unidades federativas brasileiras que possuem costa marítima.

\subsection{Dados}

\subsubsection{Fronteira e não fronteira marítima}

Neste trabalho, define-se municípios de fronteira marítima os que estão localizados na Zona Costeira Marítima (ZCM). De acordo com o Decreto o5 5300/04 (BRASIL, 2004), a ZCM é um espaço geográfico que abrange uma faixa marítima que se estende por doze milhas náuticas e uma faixa terrestre compreendida pelo limite dos municípios que fazem parte desta área.

Moraes (1999) salienta que todas as especialidades relacionadas à fronteira marítima a tornam uma localização distinta, suscetível de apropriação e utilização da mesma. De um ângulo geral, as áreas à beira mar acomodam um vasto conjunto com finalidades técnicas e meio exclusivas. Esses atributos conceituam o lugar marítimo como incomum, beneficiado de características geográficas próprias. Ademais, Carvalho (2018) define todas as atividades econômicas que recebem influência direta do mar como Economia do mar, englobando, até mesmo, as atividades que não têm o mar como matéria-prima, mas que são desempenhadas nas suas proximidades.

Conforme é mostrando na Figura 1, a ZCM é formada por dezessete estados brasileiros, sendo eles: Amapá, Pará, Maranhão, Piauí, Ceará, Rio Grande do Norte, Paraíba, Pernambuco, Alagoas, Sergipe, Bahia, Espírito Santo, Rio de Janeiro, São Paulo, Paraná, Santa Catarina e Rio Grande do Sul, com cerca de 410 municípios. 
Figura 1 - Caracterização de Zona Costeira Mapa dos Municípios da Linha de Costa do Brasil

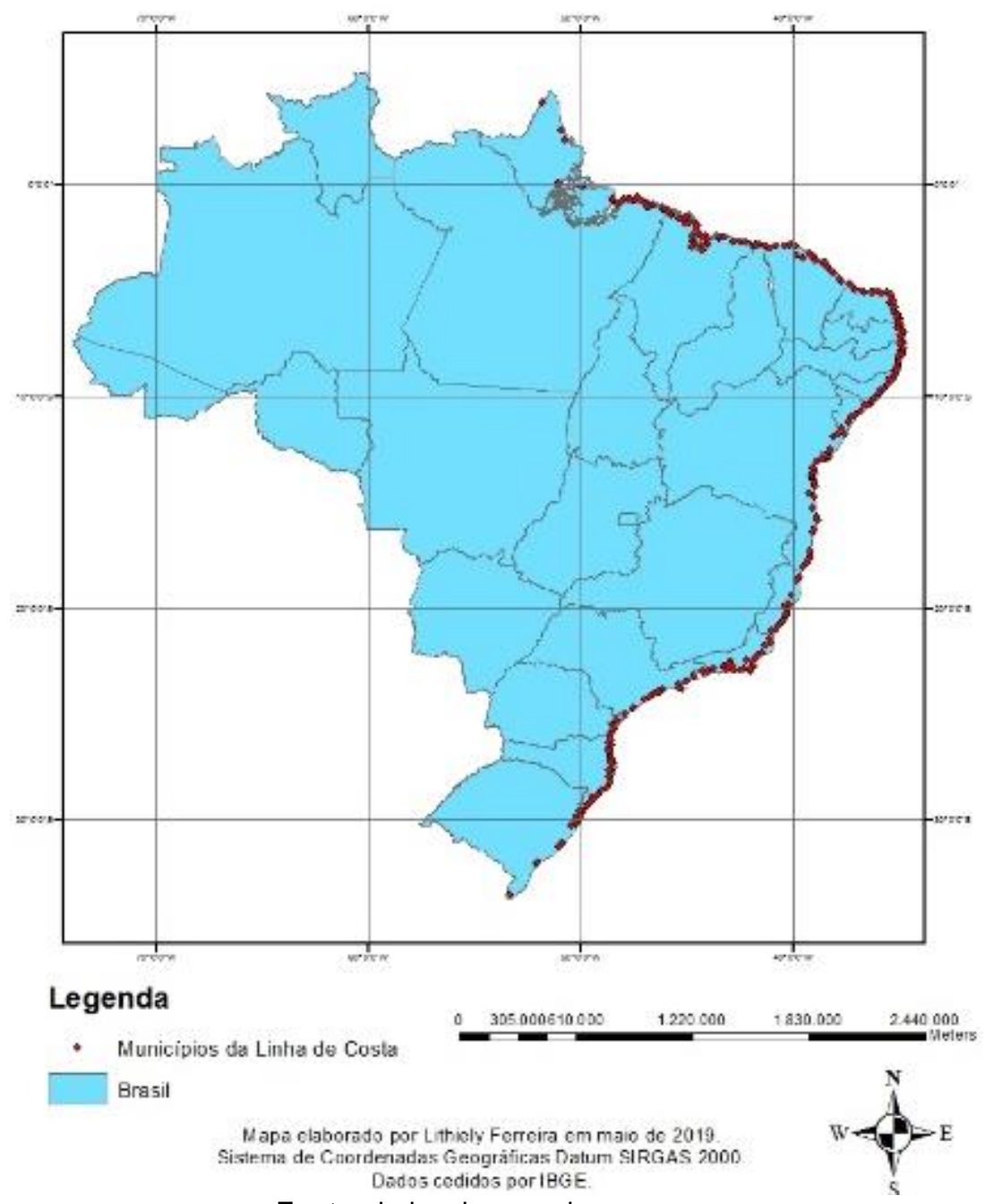

Fonte: dados da pesquisa.

\subsubsection{Descrição e natureza dos dados}

A base de dados, referente ao ano de 2010, é composta pelos 5565 municípios brasileiros, disponibilizada pelo Atlas de Desenvolvimento Humano no Brasil (ATLAS BRASIL, 2010), no qual o Programa das Nações Unidas para o Desenvolvimento no Brasil (PNUD Brasil), o Instituto de Pesquisa em Economia Aplicada (IPEA) e a Fundação João Pinheiro adaptaram a metodologia do IDH Global para calcular o IDH Municipal no Brasil, com base nos Censos Demográficos do Instituto Brasileiro de Geografia e Estatística. Também, foram utilizados dados do Departamento de Informática do Sistema Único de Saúde do Brasil DATASUS -, referentes ao ano de 2010 (DATASUS/RIPSA, 2010).

A Tabela 1 tem o intuito de apresentar as variáveis que, neste trabalho, foram consideradas as que melhor explicam a qualidade de vida das pessoas.

Tabela 1 - Indicadores do Índice de Qualidade de Vida

\begin{tabular}{|c|c|}
\hline Dimensões & Indicadores \\
\hline \multirow{6}{*}{ Ambientais } & IA1. \% da população em domicílios com coleta de lixo \\
\hline & $\begin{array}{l}\text { IA2. \% da população em domicílios com energia elétrica } \\
\text { IA3. \% da população em domicílios com abastecimento de água e esgotamento } \\
\text { sanitário inadequado }\end{array}$ \\
\hline & IE1. Índice de Gini \\
\hline & IE2. Renda per capita \\
\hline & IE3. Índice de Theil \\
\hline & IE4. População Economicamente Ativa (PEA), de 18 anos ou mais \\
\hline
\end{tabular}




\begin{tabular}{l|l}
\hline Dimensões & Indicadores \\
\hline \multirow{3}{*}{ Econômicas } & E5. \% de trabalhadores por conta própria, 18 anos ou mais \\
& IE6. \% dos ocupados no setor agropecuário, 18 anos ou mais \\
& IE7. \% dos ocupados no setor comercial, 18 anos ou mais \\
& IE8. \% dos ocupados no setor construção, 18 anos ou mais \\
& IE9. \% dos ocupados no setor extração mineral, 18 anos ou mais \\
& IE10. \% de empregados com carteira assinada, 18 anos ou mais \\
& IE11. \% de empregados sem carteira assinada, 18 anos ou mais \\
\hline IS1. Esperança de vida ao nascer \\
IS2. Taxa de fecundidade total \\
IS3. Mortalidade infantil \\
IS4. Taxa de analfabetismo de 25 a 29 anos \\
IS5. Taxa de frequência líquida ao básico \\
IS6. \% de 25 anos ou mais com superior completo \\
IS7. \% de extremamente pobres \\
IS8. População rural \\
IS9. População total \\
IS10. População total em domicílio particular permanente \\
IS11. Subíndice de escolaridade IDHM educação \\
IS12. Subíndice de frequência escolar IDHM educação \\
IS13. IDHM longevidade \\
IS14. Leitos Hospitalares
\end{tabular}

\subsubsection{Análise descritiva dos dados}

A estatística descritiva visa sumarizar e descrever qualquer grupo de dados de maneira direta. Sendo assim, para melhor entender a média dos dados utilizados neste estudo, a Tabela 2 retrata uma análise descritiva das 28 variáveis que foram estudadas. A análise apresenta a estatística da média, bem como mínimo e máximo.

Tabela 2 - Análise Descritiva das variáveis

\begin{tabular}{l|ccc}
\hline Variável & Média & Mínimo & Máximo \\
\hline IA1 & 94,04 & 0 & 100 \\
IA2 & 97,18 & 27,41 & 100 \\
IA3 & 9,2 & 0 & 85,36 \\
\hline IE1 & 0,49 & 0,28 & 0,8 \\
IE2 & 493,6 & 96,25 & 2043,74 \\
IE3 & 0,45 & 0,14 & 1,36 \\
IE4 & 0,43 & 0,14 & 0,75 \\
IE5 & 24,68 & 1,36 & 76,68 \\
IE6 & 35,55 & 0,06 & 85,12 \\
IE7 & 10,57 & 0,74 & 36,57 \\
IE8 & 6,49 & 0,14 & 26,3 \\
IE9 & 0,55 & 0 & 28,19 \\
IE10 & 30,24 & 0,9 & 83,21 \\
IE11 & 25,22 & 3,03 & 62,23 \\
\hline IS1 & 73,08 & 65,3 & 78,64 \\
IS2 & 2,19 & 1,21 & 4,89 \\
IS3 & 19,24 & 8,49 & 46,8
\end{tabular}




\begin{tabular}{l|ccc}
\hline Variável & Média & Mínimo & Máximo \\
IS4 & 6,75 & 0 & 39,89 \\
IS5 & 88,84 & 47,26 & 98,75 \\
IS6 & 5,49 & 0,28 & 33,68 \\
IS7 & 11,13 & 0 & 69,67 \\
IS8 & 5360,28 & 0 & 125336 \\
IS9 & 34277,77 & 805 & $1,13 e+07$ \\
IS10 & 34046,24 & 805 & $1,12 e+07$ \\
IS11 & 0,3966918 & 0,12 & 0,8 \\
IS12 & 0,66 & 0,26 & 0,96 \\
IS13 & 0,8 & 0,67 & 0,89 \\
IS14 & 83,22 & 0 & 29562 \\
\hline \multicolumn{4}{|c}{ Fonte: resultados da pesquisa. }
\end{tabular}

Conforme a Tabela 2, analisando as médias das variáveis, pode-se observar que, na esfera ambiental, em média, 94,04\% da população brasileira residem em domicílios com coleta de lixo; $97,18 \%$ reside em domicílios com energia elétrica e, apenas, 9,20\% residem em domicílios com abastecimento de água e esgotamento sanitário inadequado.

No âmbito econômico, observou-se que, em média, $0,43 \%$ da amostra faz parte da População Economicamente Ativa (PEA) de 18 anos ou mais; $24,68 \%$ são trabalhadores com 18 anos ou mais, que trabalham por conta própria, com renda per capita média de $\mathrm{R} \$ 493,60$.

No campo social, a expectativa de vida ao nascer apresentou média de 73,08 anos de idade; A taxa de mortalidade apontou média de 19,24 por mil habitantes e, em média, há 83,22 leitos hospitalares/ municípios no País.

\section{RESULTADOS E ANÁLISES}

Neste capítulo, serão apresentadas as estimações e discussões, estando dividida em duas seções. A primeira tratará da análise fatorial, com a estimação dos fatores que mais explicam a qualidade de vida no Brasil. Na segunda seção, serão apresentados os resultados do IQV.

\subsection{Análise Fatorial}

Primeiramente, foi efetuada uma análise fatorial de correlação a fim de verificar se as variáveis estudadas apresentam variância comum. Foi testada a confiabilidade dos fatores por meio do Critério do Alpha de Cronbach. Este método avalia se o instrumento utilizado na pesquisa consegue deduzir ou verificar aquilo que realmente se propõe, nesse caso, a criação do IQV.

O resultado de 0,92 foi satisfatório para a aplicação da análise multivariada, pois é superior ao valor recomendado pela literatura existente. Sendo assim, as escalas utilizadas, neste trabalho, são consistentes.

Posteriormente, foi testado o critério KMO (Kaiser-Meyer-Olkin), que é empregado para aferir a adequabilidade da Análise Fatorial. O teste de $\mathrm{KMO}$ deve ser maior ou igual a 0,8. Ou seja, quanto mais próximo de 1 o resultado do $\mathrm{KMO}$, mais adequado é o modelo. O resultado encontrado foi 0,8761 , tendo potencial para ser considerado um ótimo modelo.

Considerando uma variância com valores acima de um, como a literatura sugere, pode-se obter 6 fatores a partir dos 28 indicadores. Esses 6 fatores, juntos, somam $74 \%$ da variabilidade das variáveis, conforme mostra a Tabela 3.

Tabela 3 - Resultados da Análise Fatorial Exploratória

\begin{tabular}{l|ccc}
\hline \multicolumn{1}{c|}{ Fator } & Variância & Proporção & Cumulado \\
\hline Fator 1 & 8,77061 & 0,3132 & 0,3132 \\
Fator 2 & 3,26214 & 0,1165 & 0,4297 \\
Fator 3 & 3,24028 & 0,1157 & 0,5455 \\
Fator 4 & 3,15651 & 0,1127 & 0,6582 \\
Fator 5 & 1,41226 & 0,0504 & 0,7086 \\
Fator 6 & 1,03135 & 0,0368 & 0,7455 \\
\hline \multicolumn{4}{l}{ LR test: independent vs. Saturated: chi2 $(378)=2.8 \mathrm{e}+05$ Prob>chi2 $=0,0000$} \\
\hline
\end{tabular}

Fonte: resultados da pesquisa. 
Para a definição dos fatores, buscou-se analisar as cargas fatoriais acima de $0,60 \%$. No entanto, para facilitar a construção da descrição dos fatores, empregou-se a análise de cargas fatoriais de valores mais baixos.

A Tabela 4 exibe as cargas fatoriais. Notou-se que o fator 1 apresentou cargas fatoriais positivas nas variáveis IS1 (esperança de vida ao nascer), IS3 (mortalidade infantil), IS4 (taxa de analfabetismo de 25 a 29 anos), IS6 (\% de 25 anos ou mais com superior completo), IE2 (renda per capita), IE10 (\% de empregados com carteira assinada, 18 anos ou mais), IS11 (subíndice de escolaridade IDHM educação), IS12 (subíndice de frequência escolar IDHM educação), IS13 (IDHM longevidade) e cargas fatoriais negativas nas variáveis IS7 (\% de extremamente pobres) e IE11 (\% de empregados sem carteira assinada, 18 anos ou mais). Todos esses, com base no conhecimento empírico, são capazes de representar a dimensão "Ausência de Vulnerabilidade Econômica e Social".

O fator 2 apresentou cargas fatoriais positivas nas variáveis IE1 (índice de Gini), IE3 (índice de Theil), IS2 (Taxa de fecundidade total), IE7 (\% dos ocupados no setor comercial, 18 anos ou mais), IE8 (\% dos ocupados no setor construção, 18 anos ou mais) e negativo nas variáveis IA1 (\% da população em domicílios com coleta de lixo) e IA2 (\% da população em domicílios com energia elétrica). Sendo assim, esse fator tem condições de ser denominado de "Fatores de Recursos".

O fator 3 apresentou cargas fatoriais positivas nas variáveis IE7 (\% dos ocupados no setor comercial, 18 anos ou mais) e IE8 (\% dos ocupados no setor construção, 18 anos ou mais) e cargas fatoriais negativas nas variáveis IE5 (\% de trabalhadores por conta própria, 18 anos ou mais) e IE6 (\% dos ocupados no setor agropecuário, 18 anos ou mais). Desse modo, pode ser chamado de "Setores Ocupacionais Terciários".

O fator 4 mostrou cargas fatoriais positivas na variável IS9 (população total), IS10 (população total em domicílios permanentes e IS14 (leitos hospitalares) e cargas fatoriais negativas na variável IE6 (\% dos ocupados no setor agropecuário, 18 anos ou mais). Assim, pode ser definido como "Oferta de leitos hospitalares de acordo com a distribuição populacional".

O fator 5 apresentou carga fatorial positiva na variável IS5 (taxa de frequência líquida ao básico), o que o possibilita de ser identificado como "Educação básica".

O fator 6 apresentou cargas fatoriais positivas na variável IE9 (\% dos ocupados no setor extração mineral, 18 anos ou mais), porém teve um grande peso educacional nessa dimensão, sendo denominada de "Fatores Socioambientais".

Tabela 4 - Cargas fatoriais da Análise Fatorial Exploratória

\begin{tabular}{lccccccc}
\hline Variável & Fator1 & Fator2 & Fator 3 & Fator 4 & Fator 5 & Fator 6 & Comunalidade \\
\hline IS1 & 0,9111 & 0,1612 & & & 0,1005 & & 0,8766 \\
\hline IS2 & 0,5274 & 0,4029 & 0,1033 & & 0,3490 & & 0,5879 \\
\hline IS3 & 0,8994 & 0,1801 & & & 0,1048 & & 0,856 \\
\hline IS4 & 0,8124 & 0,2460 & 0,1231 & & & & 0,742 \\
\hline IS5 & & & & & 0,8114 & 0,1050 & 0,6828 \\
\hline IS6 & 0,6840 & 0,1218 & 0,4230 & 0,2253 & & & 0,723 \\
\hline IE1 & $-0,2742$ & 0,8789 & & & & & 0,8577 \\
\hline IS7 & $-0,7611$ & 0,4763 & $-0,2251$ & & $-0,1091$ & & 0,8696 \\
\hline IE2 & 0,8953 & & 0,1757 & 0,1561 & & & 0,871 \\
\hline IE3 & $-0,3097$ & 0,8482 & & & & & 0,8218 \\
\hline IE5 & 0,2122 & 0,2185 & $-0,6764$ & & 0,2894 & $-0,1193$ & 0,6493 \\
\hline IE6 & $-0,3956$ & & $-0,8458$ & $-0,1162$ & & & 0,8933 \\
\hline IE7 & 0,2395 & 0,1874 & 0,7026 & 0,1137 & 0,2022 & $-0,1436$ & 0,6605 \\
\hline IE8 & & & 0,7016 & & 0,1194 & 0,1054 & 0,5191 \\
\hline IE9 & & & & & & 0.9511 & 0,912 \\
\hline IE10 & 0,5807 & $-0,3017$ & 0,5736 & & $-0,1418$ & & 0,7862 \\
\hline IE11 & $-0,6800$ & & & & & & 0,4755 \\
\hline IA1 & 0,4092 & $-0,3922$ & 0,1483 & & & $-0,1044$ & 0,3549 \\
\hline & & & & & & &
\end{tabular}




\begin{tabular}{lccccccc}
\hline Variável & Fator1 & Fator2 & Fator 3 & Fator 4 & Fator 5 & Fator 6 & Comunalidade \\
\hline IA2 & 0,3014 & $-0,4653$ & 0,2022 & & 0,4581 & & 0.4342 \\
\hline IA3 & $-0,5871$ & 0,4365 & 0,1869 & & & & 0,5772 \\
\hline IE4 & 0,8135 & 0,3122 & 0,1225 & & 0,1913 & & 0,8145 \\
\hline IS8 & $-0,1993$ & 0,4761 & & 0,2839 & & $-0,1587$ & 0,3778 \\
\hline IS9 & & & & 0,9896 & & & 0,9879 \\
\hline IS10 & & & & 0,9895 & & & 0,9877 \\
\hline IS11 & 0,7041 & & 0,5446 & 0,1616 & 0,1161 & 0,8322 \\
\hline IS12 & 0,6818 & $-0,3809$ & 0,1041 & & 0,3598 & 0,7505 \\
\hline IS13 & 0,9112 & $-0,1610$ & & & $-0,1005$ & 0,8766 \\
\hline IS14 & & & & 0,9774 & & 0,9637
\end{tabular}

Nota: A tabela acima apresenta os resultados para valores acima de 0,10 (positivos ou negativos), para facilitar a leitura da tabela.

Fonte: resultados da pesquisa.

Sendo assim, a qualidade de vida dos municípios brasileiros pode ser explicada por seis dimensões, sendo elas: ausência de vulnerabilidade econômica e social, fatores de recursos, setores ocupacionais terciários, oferta de leitos hospitalares de acordo com a distribuição populacional, educação básica e fatores socioambientais.

\section{2 Índice de Qualidade de Vida - IQV}

Para atingir o objetivo intrínseco, foi calculado e comparado, através da Análise Fatorial Exploratória, o IQV do Brasil com o das regiões costeiras e não costeiras do País.

Conforme a Tabela 5, o Índice de Qualidade de Vida, no Brasil, foi, em média, de 41,91. Na zona costeira, o IQV, sua média é 43,79 e, na zona não costeira, o IQV, sua média, é 41,76 . Cabe salientar que o cálculo do Índice por meio da AFE pode gerar uma estimativa melhor, pois as variáveis que definem cada fator possuem maior influência. Sendo assim, no comparativo zona costeira e não costeira, o melhor IQV encontra-se na zona costeira. Apesar da evidência de um IQV melhor na Zona Costeira, o valor médio do Índice de Qualidade de Vida é baixo. Em 1988, foi promulgada a Lei Nacional de Gerenciamento Costeiro (Lei Federal no 7.661, de 16 de março de 1988), instituindo o Plano Nacional de Gerenciamento Costeiro (PNGC), tendo, entre seus objetivos, "orientar a utilização nacional dos recursos na Zona Costeira, de forma a contribuir para elevar a qualidade da vida de sua população" (BRASIL, 1988). No entanto, alguns estudos evidenciam que o PNGC ainda não foi totalmente ou parcialmente executado (e.g. ALMEIDA, 2002).

Morel (2018), utilizando uma metodologia de Propensity Score Matching (PSM), encontrou evidências contrárias aos resultados deste trabalho, em que, municípios de Zona Não Costeira apresentaram maior qualidade de vida de que os municípios de Zona Não Costeira. No entanto, Morel (2018) argumenta que esse resultado não vai de acordo com o "senso comum de que em regiões com maior potencial de concentração de atividades econômicas há maior qualidade de vida" (MOREL, 2018, p. 70). Ainda assim, ao analisar somente as características econômicas, os resultados de Morel (2018) foram de acordo com a teoria.

O teste de diferença de média entre os municípios de Zona Costeira e Zona Não Costeira apresentou estatística $t$ de $-5,41$, com 5563 graus de liberdade. O valor bicaudal correspondente é de 0,0000 , concluindo que a diferença de médias, no IQV, entre as Zonas Costeiras e Não Costeiras, é diferente de zero. O mesmo teste, considerando apenas as unidades federativas com costa marítima, demonstrou uma estatística $t$ de 6,18 com 3954 graus de liberdade e apresentou um valor bicaudal de 0,0000 , resultando, novamente, que a média no IQVA, entre as Zonas Costeiras e Não Costeiras, é diferente de zero.

Tabela 5 - Comparação da média do IQV do Brasil em relação à ZC e ZNC

\begin{tabular}{lcc} 
& Municípios & Média \\
\hline Brasil & 5565 & 41,91 \\
\hline Zona Costeira & 410 & 43,79 \\
Zona Não Costeira & 5155 & 41,76 \\
\hline
\end{tabular}

Fonte: resultados da pesquisa 
Com os valores do IQV, foi possível identificar os municípios com melhores e piores IQV na região da Zona Costeira, demonstrado na Tabela 6.

Tabela 6 - Ranking dos municípios com melhores e piores IQV - Zona Costeira

\begin{tabular}{ccc}
\hline \multirow{3}{*}{ Melhores } & Municípios costeiros & Índice \\
\cline { 2 - 3 } & Santos & 66,41 \\
\cline { 2 - 3 } & Balneário Camboriú & 70,10 \\
\cline { 2 - 3 } & Niterói & 70,73 \\
\cline { 2 - 3 } & Florianópolis & 70,80 \\
\hline \multirow{3}{*}{ Piores } & Vitória & 71,94 \\
\cline { 2 - 3 } & Roteiro & 21,06 \\
\cline { 2 - 3 } & Marcação & 25,51 \\
\cline { 2 - 3 } & Passo de Camaragibe & 26,36 \\
\cline { 2 - 3 } & Mataraca & 26,64 \\
\cline { 2 - 3 } & Água doce do Maranhão & 27,60
\end{tabular}

Fonte: resultados do estudo.

Conforme apresenta a Tabela 6, os municípios da Zona Costeira com melhores IQV são Santos - São Paulo; Balneário Camboriú - Santa Catarina; Niterói - Rio de Janeiro; Florianópolis - Santa Catarina e Vitória - Espírito Santo. Esses municípios estão localizados na região sudeste e sul do Brasil e são grandes municípios e com grande atividade econômica. Santos, por exemplo, abriga o maior porto da América Latina; Balneário Camboriú é uma cidade turística; Niterói foi capital do Estado do Rio de Janeiro até 1974 e, atualmente, é um dos principais centros comerciais e industriais do Estado, e Florianópolis e Vitória são as capitas de seus Estados.

Já os municípios com piores IQV na Zona Costeira foram Roteiro - Alagoas; Marcação - Paraíba; Passo de Camaragibe - Alagoas; Mataraca - Paraíba e o município de Água doce do Maranhão - Maranhão. Observa-se que estes municípios estão localizados na região nordeste do Brasil e são pequenos municípios com uma população que varia, aproximadamente, de 6 mil a 14 mil habitantes.

No que se refere a todos os municípios brasileiros, a Tabela 7 representa a escala do IQV, que, felizmente, não se aplica a nenhum município com IQV péssimo. Um IQV razoável, em média de 40,77, está presente em 2.269 municípios. Um IQV bom, 58,44\%, está presente em 3.252 municípios brasileiros. Um IQV ótimo, 0,79\%, está presente em apenas 44 municípios. E nenhum município brasileiro possui IQV excelente.

Tabela 7 - Escala IQV

\begin{tabular}{|c|c|c|c|}
\hline Escala IQV & Obs. & $\%$ & Cum. \\
\hline Péssimo & 0 & 0 & 0 \\
\hline Razoável & 2.269 & 40,77 & 40,77 \\
\hline Bom & 3.252 & 58,44 & 99,21 \\
\hline Ótimo & 44 & 0,79 & 100 \\
\hline Excelente & 0 & 0 & 100 \\
\hline Total & 5.565 & 100 & \\
\hline
\end{tabular}

A Tabela 8 expõe o IQV por regiões demográficas, o que permite distinguir as regiões com maiores valores daquelas com menores valores.

Dentre as regiões com os melhores valores, destacam-se: o Distrito Federal, com 64,95; Santa Catarina, com 48,23 e Rio de Janeiro, com 47,74. Tais valores, possivelmente, podem ser justificados pelas seguintes causas: o primeiro, por referir-se à região que contém a capital federativa do Brasil; o segundo, por se tratar de uma região que apresenta um forte e diversificado setor industrial, e o terceiro, por referir-se ao estado que contém a segunda maior metrópole do País.

Dentre as regiões com os piores valores, destacam-se: Alagoas com 32,00; Paraíba, com 33,64 e Piauí, com 34,64 . Tais valores podem ser justificados pelo fato de a economia de ambos ser fortemente dependente do setor primário. 
Tabela 8 - IQV por Regiões Demográficas

\begin{tabular}{|c|c|c|c|}
\hline Região Demográfica & Unidades federativas & Obs & Média \\
\hline \multirow[t]{7}{*}{ Norte } & Rondônia & 52 & 43,31 \\
\hline & Acre & 22 & 40,00 \\
\hline & Amazonas & 62 & 40,36 \\
\hline & Roraima & 15 & 44,48 \\
\hline & Pará & 143 & 40,59 \\
\hline & Amapá & 16 & 46,18 \\
\hline & Tocantins & 139 & 41,39 \\
\hline \multirow[t]{9}{*}{ Nordeste } & Maranhão & 217 & 35,46 \\
\hline & Piauí & 224 & 34,64 \\
\hline & Ceará & 184 & 36,78 \\
\hline & Rio Grande do Norte & 167 & 35,91 \\
\hline & Paraíba & 223 & 33,64 \\
\hline & Pernambuco & 185 & 35,73 \\
\hline & Alagoas & 102 & 32,00 \\
\hline & Sergipe & 75 & 35,84 \\
\hline & Bahia & 417 & 36,14 \\
\hline \multirow[t]{4}{*}{ Sudeste } & Minas Gerais & 853 & 42,29 \\
\hline & Espírito Santo & 78 & 45,65 \\
\hline & Rio de Janeiro & 92 & 47,74 \\
\hline & São Paulo & 645 & 47,54 \\
\hline \multirow[t]{3}{*}{ Sul } & Paraná & 399 & 45,14 \\
\hline & Santa Catarina & 293 & 48,23 \\
\hline & Rio Grande do Sul & 496 & 47,61 \\
\hline \multirow[t]{4}{*}{ Centro-oeste } & Mato Grosso do Sul & 78 & 45,75 \\
\hline & Mato Grosso & 141 & 44,36 \\
\hline & Goiás & 246 & 44,24 \\
\hline & Distrito Federal & 1 & 64,95 \\
\hline
\end{tabular}

Fonte: resultados da pesquisa.

Um mapa de calor do Índice de Qualidade de Vida por município é apresentado na Figura 2, em que municípios, com cores mais quentes, têm melhor nível de IQV, e municípios, com cores mais frias, possuem pior nível de IQV. 
Figura 2 - Mapa dos municípios brasileiros de acordo com o Índice de Qualidade de Vida (IQV)

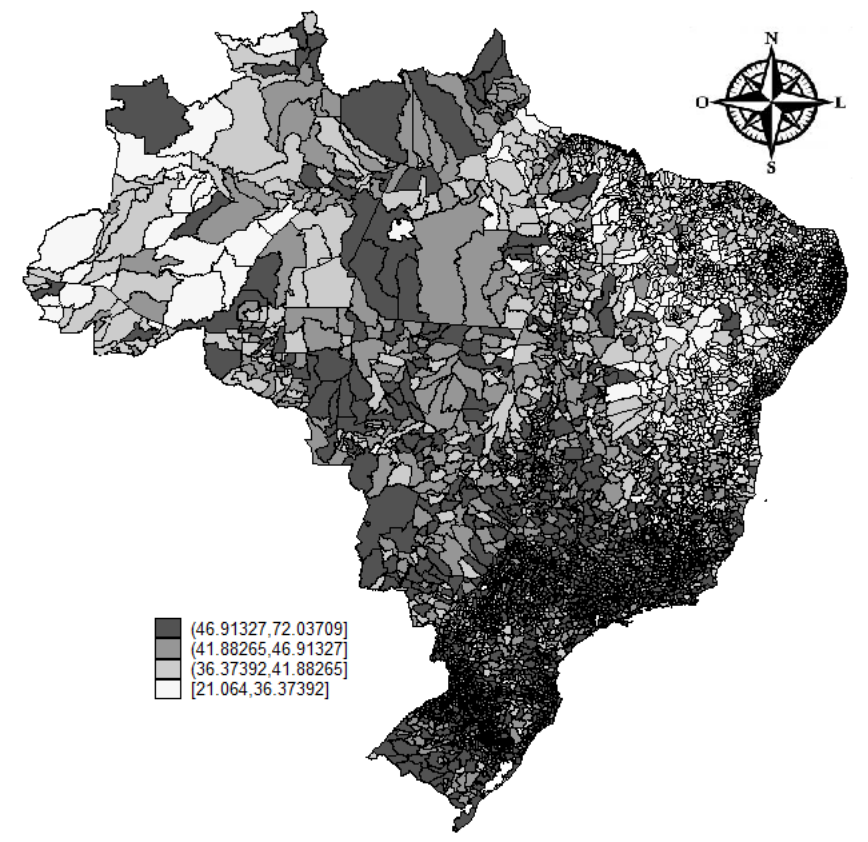

Fonte: Resultados da pesquisa.

A Figura 3 apresenta, através de um mapa de calor, a qualidade de vida por estado brasileiro. As cores mais quentes indicam estados com maior nível de IQV, e as cores mais suaves indicam estados com menor nível de IQV.

Figura 3 - Mapa do Índice de Qualidade de Vida (IQV) dos estados brasileiros

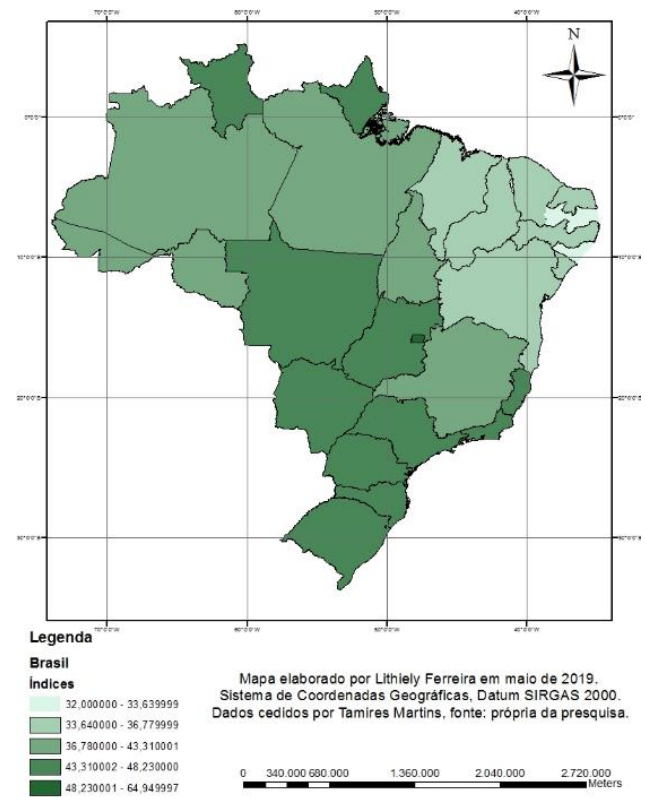

Fonte: resultados da pesquisa.

Averígua-se que as regiões nordeste e norte do Brasil possuem, em média, uma menor qualidade de vida, e as regiões sudeste, centro-oeste e sul apresentam melhor qualidade de vida. Almeida (2002) analisou a qualidade de vida da comunidade de Prainha do Canto Verde da cidade de Beberibe no Estado do Ceará (região nordeste) e evidenciou, como necessidades mais carentes, a alimentação e a organização comunitária, e, como mais satisfatórias, a saúde e a energia. 


\section{CONSIDERAÇÕES FINAIS}

A avaliação da qualidade de vida é um dos grandes desafios para as ciências econômicas nos tempos atuais. Em grande medida, isso se deve à própria dificuldade em conceituar o termo "qualidade de vida". Nesse contexto, a motivação deste estudo se deu pela constatação da lacuna existente na literatura econômica brasileira em relação a trabalhos que considerem os níveis de bem-estar dos indivíduos associado ao perfil socioeconômico deles.

Sendo assim, o presente estudo buscou criar o Índice de Qualidade de Vida (IQV), com o objetivo de averiguar a qualidade de vida no Brasil, nos municípios em geral e comparar os 5155 municípios da Zona não costeira com os 410 que compõem a Zona costeira. Para isso, utilizaram-se dados obtidos do Censo, do DATASUS/RIPSA e do Atlas do Desenvolvimento Humano referentes ao ano de 2010.

Com base no conhecimento empírico, foram selecionadas 28 variáveis, sendo essas, posteriormente, organizadas em três dimensões: ambiental, econômica e social. Através da utilização da Análise Multivariada com técnica de Análise Fatorial, surgiram seis dimensões latentes que explicam $74 \%$ da variabilidade dos indicadores.

Por meio dos escores fatorais dessas dimensões, o estudo obteve os seguintes níveis de IQV para o Brasil, para a Zona Costeira e para a Zona não costeira: 41,91; 43,79 e 41,76, respectivamente. O estudo, também, obteve o ranking dos cinco melhores e dos cinco piores municípios de acordo com o IQV. Melhores: São Caetano do Sul $(72,03)$, Vitória $(71,94)$, Nova Lima $(71,39)$, Florianópolis $(70,80)$ e Niterói $(70,73)$. Piores: Roteiro $(21,06)$, Branquinha $(21,53)$, Cuité de Mamanguape $(22,15)$, Olivença $(23,03)$ e São José do Piauí $(24,18)$.

Por esse método, identificou-se que a melhor qualidade de vida se encontra na Zona Costeira apesar de não haver grande discrepância com relação à Zona não costeira. Identificou-se que os municípios com melhor qualidade de vida se encontram nas regiões sudeste e sul. Em contrapartida, os municípios com pior qualidade de vida se encontram na região nordeste do País.

Considerando os resultados obtidos, espera-se que a adoção do IQV colabore no sentido de tornar as políticas públicas mais direcionadas no que tange à melhoria dos indicadores ambientais, econômicos e sociais. Espera-se que, a longo prazo, essa medida promova em aumento efetivo e consistente dos níveis de IQV, de modo que, futuramente, mais municípios possam ser classificados como "bons" e, aqueles que já se encontram nesse nível, possam tornar-se "excelentes".

Por fim, almeja-se que o presente trabalho possa colaborar com o desenvolvimento de futuras pesquisas que abordem o tema a qualidade de vida, como, por exemplo, aquelas que consideram as possíveis causas históricas e políticas que justifiquem as discrepâncias entre os dados obtidos para cada município.

\section{REFERÊNCIAS}

ALMEIDA, Henrique Luís de Paula. Indicadores de qualidade de vida instrumento para o monitoramento participativo da qualidade de vida de comunidades costeiras tradicionais: o caso da Prainha do Canto Verde, Beberibe/CE. Dissertação (Mestrado em Desenvolvimento e Meio Ambiente) - Universidade Federal do Ceará. 2002.

ATLAS BRASIL - ATLAS DE DESENVOLVIMENTO HUMANO DO BRASIL. 2010. Disponível em: < $<$ http://www.atlasbrasil.org.br/2013/pt/o atlas/idhm/> Acessado em: 18 de setembro de 2018.

BRASIL. Lei no 7.661, de 16 de maio de 1988. Regulamento Institui o Plano Nacional de Gerenciamento Costeiro e dá $\begin{array}{lllll}\text { outras } & \text { providências. } & \text { Disponível } & \text { em: }\end{array}$ http://www.planalto.gov.br/ccivil 03/leis/l7661.htm\#: :text=Institui\%200\%20Plano\%20Nacional\%20de,Art.>. Acesso em: 23/12/2020.

BRASIL. A Zona Costeira e seus múltiplos usos. Breve histórico sobre motivos de ocupação. 2019. Disponível em: < https://www.mma.gov.br/gestao-territorial/gerenciamento-costeiro/zona-costeira-e-seus-m\%C3\%BAltiplos-usos >. Acesso em: 10/06/2019.

BRASIL. Decreto № 5.300 de 7 de dezembro de 2004. Regulamenta a Lei no 7.661, de 16 de maio de 1988, que institui o Plano Nacional de Gerenciamento Costeiro - PNGC, dispõe sobre regras de uso e ocupação da zona costeira e estabelece critérios de gestão da orla marítima, e dá outras providências. 2004.

BLOMQUIST, Glenn C.; BERGER, Mark C.; HOEHN, John P. New estimates of quality of life in urban areas. The American Economic Review, p. 89-107, 1988.

CARVALHO, A. B. Economia do Mar: Valor, conceito e importância para o Brasil. 2018. 200 fls, Trabalho de Conclusão de Doutorado (Tese). Curso de Economia, Pontifícia Universidade Católica do Rio Grande do Sul PUC-RS. Porto Alegre, 2018.

DATASUS/RIPSA. Departamento de informática do Sistema Único de Saúde do Brasil - DATASUS, Rede Interagencial de Informações para a Saúde - RIPSA. Disponível em: <http://www.ripsa.org.br/>. Acesso em: 02/04/2019,

DISTASO, Alba. Well-being and/or quality of life in EU countries through a multidimensional index of sustainability. Ecological Economics, v. 64, n. 1, p. 163-180, 2007. 
FLECK, M.P.A.; LOUZADA, S.; XAVIER, M.; CHACHAMOVICH, E.; VIEIRA, G.; SANTOS, L.; PINZON, V. Aplicação da versão em português do instrumento de avaliação de qualidade de vida da Organização Mundial da Saúde (WHOQOL100). Revista de Saúde Pública, Rio de Janeiro, v.33, n.2, p.198-205, 1999.

GONÇALVES, Aguinaldo; VILARTA, Roberto Qualidade de Vida: identidades e indicadores. In: GONÇALVES, Aguinaldo e VILARTA, Roberto (orgs.). Qualidade de Vida e atividade física: explorando teorias e práticas. Barueri: Manole, 2004, p.03-25.

HAIR JR., J.F.; WILLIAM, B.; BABIN, B.; ANDERSON, R.E. Análise multivariada de dados. 6.ed. Porto Alegre: Bookman, 2009.

HENDERSON, J. Vernon et al. The global distribution of economic activity: nature, history, and the role of trade. The Quarterly Journal of Economics, v. 133, n. 1, p. 357-406, 2018.

HERCULANO, Selene C. A qualidade de vida e seus indicadores. Ambiente e Sociedade, v. 1, n. 2, p. 77-99, 1998.

KRAN, Faida; FERREIRA, Frederico Poley Martins. Qualidade de vida na cidade de Palmas-TO: uma análise através de indicadores habitacionais e ambientais urbanos. Ambiente \& Sociedade, v. 9, n. 2, p. 123-141, 2006.

KRUGER, Rayssa Vieira; DE OLIVEIRA, Sibele Vasconcelos; DOS SANTOS, Priscila Soares. Análise multidimensional da qualidade de vida nos municípios do Rio Grande do Sul (2010). Economia e Desenvolvimento, v. 32, n. Esp., p. 3, 2020.

LAMBERT, J. G.; HALL, C. A.; BALOGH, S.; GUPTA, A.; e Arnold, M. Energy, EROI and quality of life. Energy Policy, v. 64, p. 153-167, 2014.

LIMA, J. E. Curso de Análise Estatística Multivariada. Apostila. Departamento de Economia Rural. Universidade Federal de Viçosa. 2017.

MIDGLEY, J. Social Development: The Developmental Perspective in Social Welfare. London - Thousand Oaks New Delhi: SAGE Publications, 1995.

MORAES, A. C. R. Contribuições Para a Gestão da Zona Costeira do Brasil. Elementos para uma Geografia do Litoral Brasileiro. São Paulo: Hucitec/Edusp, p. 27 - 56, 1999.

MINGOTI, S. A. Análise de dados através de métodos de estatística multivariada. Uma abordagem aplicada. Editora UFMG. Belo Horizonte. 2013.

MOREL, Blanca Lila Gamarra. Desenvolvimento econômico e qualidade de vida no Brasil: uma análise comparativa entre os municípios costeiros e os municípios não costeiros. Tese (Doutorado em Economia do desenvolvimento) Pontifícia Universidade Católica do Rio Grande do Sul. 2018.

NAHAS, Maria Inês Pedrosa et al. Metodologia de construção de índices e indicadores sociais como instrumentos balizadores da gestão municipal da qualidade de vida urbana: uma síntese da experiência de Belo Horizonte. Migração e ambiente nas aglomerações urbanas. Campinas: Núcleo de Estudos de População/Unicamp, v. 465, p. 487, 2001.

NUSSBAUM, M. C.; SEN, A. K. La calidad de vida. Traducción de Roberto Ramón Reyes Mazzoni. México: FCE, 1996. ONU. Organização das Nações Unidas. Relatório de Desenvolvimento Humano Global, 2013.

PNUD - Programa das Nações Unidas para o Desenvolvimento. Relatório de Desenvolvimento Humano 1990.1990. Disponível em: <http://hdr.undp.org/en/reports/global/hdr1990>. Acesso em: 20/03/2019.

PNUD - Programa das Nações Unidas para o Desenvolvimento. Relatório de Desenvolvimento Humano 2010.2010. Disponível em: <https://www.br.undp.org/content/brazil/pt/home/library/idh/relatorios-de-desenvolvimentohumano/relatorio-do-desenvolvimento-humano-200010.html >. Acesso em: 20/03/2019.

PNUD - Programa das Nações Unidas para o Desenvolvimento. Relatório do Desenvolvimento Humano 2015.0 Trabalho como Motor do Desenvolvimento Humano. 2015. Disponível em: < https://www.br.undp.org/content/brazil/pt/home/idh0/relatorios-de-desenvolvimento-humano/rdhs-globais.html\#2014>.

Acesso em: 20/03/2019.

PNUD - Programa das Nações Unidas para o Desenvolvimento. Progresso Multidimensional: o bem-estar para além da renda. Relatório de Desenvolvimento Humano Regional para a América Latina e o Caribe. 2016. Disponível em: < https://www.undp.org/>. Acesso em: 20/04/2019.

PNUD - Programa das Nações Unidas para o Desenvolvimento. Relatório do Desenvolvimento Humano 2016. Desenvolvimento humano para todos. $2017 . \quad$ Disponível em: https://www.br.undp.org/content/brazil/pt/home/idh0/relatorios-de-desenvolvimento-humano/rdhs-globais.html\#2014>. Acesso em: 20/03/2019.

RAPPAPORT, Jordan; SACHS, Jeffrey D. The United States as a coastal nation. Journal of Economic growth, v. 8, n. 1 , p. 5-46, 2003.

SEN, Amartya. Development as Capability Expansion, Jounal of Development Planning, n. 19, 1989.

SLOTTJE, Daniel J. Measuring the quality of life across countries. The Review of economics and statistics, p. 684-693, 1991.

VIANA, Lenival Santiago et al. Qualidade de vida no meio rural brasileiro: o caso do sertão de Alagoas. Revista de Economia e Sociologia Rural, v. 18, n. 2, p. 181-204, 2019. 
